\title{
Pathfollowing for Essentially Singular Boundary Value Problems with Application to the Complex Ginzburg-Landau Equation *
}

\author{
Georg Kitzhofer ${ }^{\mathrm{a}}$ Othmar Koch ${ }^{\mathrm{b}}$ Ewa Weinmüller ${ }^{\mathrm{a}, *}$ \\ ${ }^{a}$ Vienna University of Technology, Institute for Analysis and Scientific \\ Computing, Wiedner Hauptstrasse 8-10, A-1040 Wien, Austria \\ ${ }^{\mathrm{b}}$ University of Tübingen, Department of Mathematics, Auf der Morgenstelle 10, \\ D-72076 Tübingen, Germany
}

\begin{abstract}
We discuss a pathfollowing strategy based on pseudo-arclength parametrization for the solution of parameter-dependent boundary value problems for ordinary differential equations. We formulate criteria which ensure the successful application of this method for the computation of solution branches with turning points for problems with an essential singularity. Finally, we demonstrate that a Matlab implementation of the solution method based on an adaptive collocation scheme is well suited to solve problems of practical relevance. As one example, we compute solution branches for the complex Ginzburg-Landau equation which start from multi-bump solutions of the nonlinear Schrödinger equation. Following the branches around turning points, real-valued solutions of the nonlinear Schrödinger equation can easily be computed.
\end{abstract}

Key words: Boundary value problems for ordinary differential equations, essential singularity, pathfollowing strategy, pseudo-arclength parametrization, turning point, nonlinear Schrödinger equation, complex Ginzburg-Landau equation, self-similar blow-up solutions, collocation methods

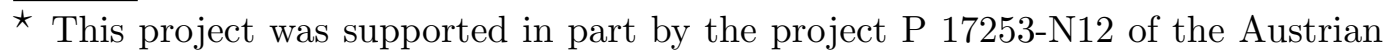
Science Fund FWF and the Special Research Program SFB 382 at the University of Tübingen, Germany.

* Corresponding author.

Email addresses: g.kitzhofer@kabsi.at (Georg Kitzhofer), othmar@othmar-koch.org (Othmar Koch), e.weinmueller@tuwien.ac .at (Ewa Weinmüller).

URLs: www.othmar-koch.org (Othmar Koch), www.math.tuwien.ac.at/ ewa (Ewa Weinmüller).

Preprint submitted to Elsevier Science 7 April 2006 


\section{Introduction}

In this paper, we consider parameter-dependent singular boundary value problems for ordinary differential equations of the form

$$
\begin{aligned}
& z^{\prime}(t)=\frac{1}{t^{\alpha}} f(t, z(t) ; \lambda), \quad t \in(0,1], \\
& b(z(0), z(1))=0
\end{aligned}
$$

where the case $\alpha>1$ in (1) represents an essential singularity. Motivated by applications, we will also incorporate problems with a singularity of the first kind, where $\alpha=1$, into our treatment. We are interested in computing sets of solutions in dependence of the known (real) parameter $\lambda$. The problems in our focus typically arise from the transformation of problems on an unbounded domain to the interval $[0,1]$. In applications, these problems are often encountered in the computation of self-similar blow-up solutions of nonlinear partial differential equations. A collection of typical problems can be found in [1] together with a discussion of numerical solution methods for fixed parameter values. In contrast, here we consider a pathfollowing method which can serve to compute branches of solutions in dependence of the parameter. The algorithm is based on pseudo-arclength parametrization and is thus even suitable for solution branches with turning points. To solve the boundary value problems occurring in the course of the pathfollowing algorithm, we use polynomial collocation on grids that are chosen according to an adaptive mesh selection strategy based on the equidistribution of the global error [2].

The paper is organized as follows: In $\S 2$ we recapitulate the analytical properties of (1), (2) which are necessary to formulate conditions ensuring that our pathfollowing strategy is well-defined. Proofs for these preliminary considerations can be found in [3], [4]. In $\S 3$, we describe our pathfollowing strategy and formulate the conditions for the problem (1), (2) which - according to general results given in [5] for parameter-dependent problems in Banach spaces ensure the well-posedness of our solution method, see also [6]. In $\S 4$, we give a numerical example illustrating the performance of a Matlab implementation of our procedure. Finally, in $\S 5$ we demonstrate the success of the strategy for a practically relevant problem: Blow-up solution profiles of the complex Ginzburg-Landau equation (CGL), which is a perturbation of the celebrated nonlinear Schrödinger equation (NLS), can be computed from an ODE problem on an unbounded domain after performing a similarity reduction [7]. The resulting problem can be transformed to our present problem class. We show that the transformed problem is a well-posed boundary value problem with an essential singularity, and the boundary conditions are admissible according to the analytical results given in [3]. Thus, our adaptive collocation solver can be used reliably to perform our pathfollowing algorithm. We demonstrate that 
solution branches starting from (unstable) multi-bump solutions of the NLS [8] can be computed across turning points, yielding a set of stable solutions of the CGL. The branches finally terminate at real solutions of the NLS.

\section{Analytical Properties of Problems with an Essential Singularity}

In this section, we provide the analytical prerequisites for the discussion of pathfollowing in $\S 3$. To give a general framework encompassing the majority of practically relevant problems, cf. $\S \S 4-5$, we consider nonlinear systems of the form

$$
\begin{aligned}
& T(t) z^{\prime}(t)=f(t, z(t)), \quad t \in(0,1], \\
& z \in C[0,1] \cap C^{1}(0,1], \\
& b(z(0), z(1))=0,
\end{aligned}
$$

where $z$ is a vector-valued function of dimension $n, b: \mathbb{R}^{n} \times \mathbb{R}^{n} \rightarrow \mathbb{R}^{p}$,

$$
T(t):=\operatorname{diag}\left(t^{\alpha_{1}} I_{1}, t^{\alpha_{2}} I_{2}, \ldots, t^{\alpha_{r}} I_{r}\right),
$$

and the $I_{k}$ are unit matrices with either $\alpha_{k} \geq 1$ for $1 \leq k \leq r$, or $\alpha_{k} \geq 1$ for $1 \leq k \leq r-1$ and $\alpha_{r}=0$. It will turn out that (4) is equivalent to $n-p$ linearly independent constraints on $z(0)$, and (5) should provide $p$ additional conditions to ensure local uniqueness of the solution.

As a first step in the analysis of (3)-(5) we will examine the solution structure of linear systems

$$
\begin{aligned}
& T(t) z^{\prime}(t)=(M+A(t)) z(t)+g(t), \quad t \in(0,1], \\
& z \in C[0,1] \cap C^{1}(0,1] \\
& B_{0} z(0)+B_{1} z(1)=\gamma,
\end{aligned}
$$

where the matrix $M \in \mathbb{R}^{n \times n}$ is a block upper triangular matrix,

$$
M:=\left(\begin{array}{cccc}
M_{11} & M_{12} & \ldots & M_{1 r} \\
0 & M_{22} & \ldots & M_{2 r} \\
\vdots & \vdots & \ldots & \vdots \\
0 & 0 & \ldots & M_{r r}
\end{array}\right)
$$


and $A, g \in C[0,1]$. Each matrix $M_{k k}$ is a square matrix of the same size as $I_{k}$ which is assumed to be nonsingular when $\alpha_{k} \neq 0$ and has no eigenvalues that are purely imaginary. When $\alpha_{r}=0$ then $M_{r r}=0$. Moreover, $B_{0}, B_{1} \in \mathbb{R}^{p \times n}$ are constant matrices and $\gamma \in \mathbb{R}^{p}$ is a constant vector. In general, $p \leq n$.

The analytical properties of (3)-(5) and (7)-(9) have been discussed in full detail in [3], [4]. The analysis when a singularity of the first kind is present, which is incorporated into the treatment in [3], has initially been established in [9]. In these papers the Fredholm theory for linear systems has been established and existence and smoothness results for nonlinear problems have been provided. In this section we recapitulate the most important of these fundamental results.

\subsection{Linear systems}

Initially, we examine the system

$$
T(t) z^{\prime}(t)=M z(t)+g(t), \quad 0<t \leq 1
$$

with matrices $T(t)$ and $M$ defined in (6) and (10), respectively. Let

$$
\begin{aligned}
& M=D+U, \quad D=\operatorname{diag}\left(M_{11}, M_{22}, \ldots, M_{r r}\right), \\
& Z(t)=\operatorname{diag}\left(Z_{1}(t), \ldots, Z_{r}(t)\right), \\
& P=\operatorname{diag}\left(P_{1}, \ldots, P_{r}\right), \\
& Q=\operatorname{diag}\left(Q_{1}, \ldots, Q_{r}\right),
\end{aligned}
$$

with

$$
Z_{k}(t)= \begin{cases}\exp \left[M_{k k}\left(\delta^{1-\alpha_{k}}-t^{1-\alpha_{k}}\right) /\left(\alpha_{k}-1\right)\right], & \alpha_{k} \neq 1 \\ \exp \left[\log (t / \delta) M_{k k}\right], & \alpha_{k}=1\end{cases}
$$

Note that $Z_{k}(t)$ is the fundamental solution matrix satisfying

$$
t^{\alpha_{k}} Z_{k}^{\prime}(t)-M_{k k} Z_{k}(t)=0, \quad 0<t \leq 1, \quad Z_{k}(\delta)=I_{k},
$$

for $0<\delta \leq 1$. If $\alpha_{k} \neq 0, P_{k}, Q_{k}$ are defined by

$$
Q_{k}:=\frac{1}{2 \pi \mathrm{i}} \int_{\Gamma_{k}^{-}}\left(\lambda I_{k}-M_{k k}\right)^{-1} d \lambda
$$




$$
P:=\frac{1}{2 \pi \mathrm{i}} \int_{\Gamma_{k}^{+}}\left(\lambda I_{k}-M_{k k}\right)^{-1} d \lambda
$$

where $\Gamma_{k}^{-}$and $\Gamma_{k}^{+}$denote closed contours (oriented canonically) in the left and right complex halfplane, respectively, such that each eigenvalue of $M$ is enclosed by either $\Gamma_{k}^{-}$or $\Gamma_{k}^{+}$. The matrices $Q_{k}$ and $P_{k}$ are projections onto the invariant subspaces of $M_{k k}$ associated with the eigenvalues having negative and positive real parts, respectively. We set $P_{k}=Q_{k}=0$ if $\alpha_{k}=0$. Furthermore, define

$$
R:=I-P-Q= \begin{cases}\operatorname{diag}\left(0, \ldots, 0, I_{r}\right), & \alpha_{r}=0 \\ 0, & \alpha_{r} \neq 0 .\end{cases}
$$

Finally, define for $0<\delta \leq 1$

$$
\begin{aligned}
(\mathcal{B} g)(t):= & Z(t) \int_{0}^{t} Q Z^{-1}(s) T^{-1}(s) g(s) d s \\
& +Z(t) \int_{\delta}^{t} P Z^{-1}(s) T^{-1}(s) g(s) d s+R \int_{\delta}^{t} g(s) d s .
\end{aligned}
$$

Lemma 1 Let $g \in C[0,1]$. Then any continuous solution of (11) must satisfy

$$
z(t)=Z(t)[P z(\delta)+R z(\delta)]+(\mathcal{B}[U z+g])(t) .
$$

The most general linear equation that we consider is (7), (8), where $A, g \in$ $C[0,1]$ and

$$
(I-R) A(0)=0
$$

In order to discuss the existence and uniqueness of the solution of (7), (8), a contraction argument for the operator defined in (14) is used. Consequently, the following result holds: Let $p=\operatorname{rank}[P+R]$, W be an $n \times p$ matrix which consists of linearly independent columns of $(P+R)$, and define

$$
X(t):=Y(t) W
$$

where $Y(t)$ is the unique solution of

$$
\begin{aligned}
& T(t) Y^{\prime}(t)=(M+A(t)) Y(t), \quad 0<t \leq 1, \\
& Y(t) \in C[0,1] \cap C^{1}(0,1], \\
& R Y(\delta)=R, \quad P Y(\delta)=P .
\end{aligned}
$$


In addition, let $\tilde{y}$ be the unique particular solution of $(7)$, (8) subject to the boundary conditions

$$
R \tilde{y}(\delta)=P \tilde{y}(\delta)=0
$$

Then we have

Theorem 2 Any solution of (7), (8) has the form

$$
z(t)=X(t) \beta+\tilde{y}(t)
$$

with a unique $\beta \in \mathbb{R}^{p}$.

We now answer the question under which circumstances the solution of (7), (8) given by (17) satisfies the boundary conditions (9). Our aim is to establish conditions on $B_{0}$ and $B_{1}$ which lead to a Fredholm alternative for (7)-(9). To do this it is convenient to introduce the differential expression

$$
l(z)=T z^{\prime}-(M+A) z
$$

and associate with it the operator defined by

$$
\mathcal{L} z=l(z)
$$

for

$$
z \in \mathcal{D}=\left\{z \in C[0,1]: T z^{\prime} \in C[0,1], B_{0} z(0)+B_{1} z(1)=0\right\} .
$$

Then, $\mathcal{D}$ together with the norm

$$
\|z\|_{\mathcal{L}}:=\|z\|_{\infty}+\|\mathcal{L} z\|_{\infty}
$$

is a Banach space, and we can prove:

\section{Theorem 3 If}

$$
\operatorname{rank}\left[B_{0}, B_{1}\right]=k
$$

then $\mathcal{L}$ is Fredholm ${ }^{1}$ with index $p-k$. Furthermore, if $\mathcal{L}^{-1}$ exists, it is bounded.

In applications we are primarily interested in the case when $\mathcal{L}$ is Fredholm with index 0 . We therefore assume that $B_{0}, B_{1}$ are $p \times n$ matrices, $\gamma \in \mathbb{R}^{p}$, and that (20) holds with $k=p$. On substitution of (17) into (9) we find

$\overline{1}$ A linear operator $\mathcal{L}$ is said to be Fredholm with index $q$ if it is a bounded operator mapping a Banach space $Y$ into a Banach space $Z$, the image of $\mathcal{L}$ is closed, and the nullspace of $\mathcal{L}$ has finite dimension $q$. 
Theorem 4 The problem (7)-(9) has a unique solution for all $g \in C[0,1]$ and $\gamma \in \mathbb{R}^{p}$ if and only if the $p \times p$ matrix $\left[B_{0} X(0)+B_{1} X(1)\right]$ is nonsingular.

Remark 5 Clearly, the restriction that the solution be continuous at $t=0$ is not useful when constructing numerical schemes. It turns out that the relation

$$
Q z(0)=Q(M+R)^{-1}((R-I) g(0)+R z(0))
$$

is more satisfactory. Equations (9) and (21) are the $n$ linearly independent boundary conditions which must be employed when the equation (7) is solved in practice. This and other related questions are also discussed in [4].

\subsection{Nonlinear case}

We now formulate smoothness results for nonlinear problems (3)-(5). We first make a number of assumptions.

(N1) Problem (3)-(5) has a solution $z(t)$. With this solution and some $\rho>0$ we associate the spheres

$$
S_{\rho}(z(t)):=\left\{y \in \mathbb{R}^{n}:|z(t)-y| \leq \rho\right\}
$$

and the tube

$$
T_{\rho}(z):=\left\{(t, y): t \in[0,1], y \in S_{\rho}(z(t))\right\}
$$

(N2) For some $\rho>0, f(t, z(t))$ is continuously differentiable with respect to $z$, and $\frac{\partial f}{\partial z}(t, z)$ is continuous on $T_{\rho}(z)$.

(N3) For all $y \in S_{\rho}(z(0))$, the matrix

$$
M(y):=\frac{\partial f}{\partial z}(0, y)
$$

has the fixed block upper triangular structure introduced in (10). In addition, the matrix

$$
M:=M(z(0))
$$

satisfies all the conditions concerning the matrix $M$ introduced earlier.

(N4) $b(v, w)$ is a vector-valued function of dimension

$$
p:=\operatorname{rank}[P+R]
$$

which is continuously differentiable on $S_{\rho}(z(0)) \times S_{\rho}(z(1))$. 
(N5) The solution $z(t)$ is isolated. This means that the linearized problem

$$
\begin{aligned}
& T(t) u^{\prime}(t)-G(t) u(t)=0, \\
& u \in C[0,1] \cap C^{1}(0,1], \\
& B_{0} u(0)+B_{1} u(1)=0
\end{aligned}
$$

with

$$
G(t)=\frac{\partial f}{\partial z}(t, z(t)), \quad B_{0}=\frac{\partial b}{\partial v}(z(0), z(1)), \quad B_{1}=\frac{\partial b}{\partial w}(z(0), z(1))
$$

has only the trivial solution.

The requirement that the solution of (3) be continuous at $t=0$ obviously implies the following restriction on the solution:

$$
(I-R) f(0, z(0))=0 .
$$

This immediately yields

Lemma 6 Let $z$ satisfy (3) and (4). Then $T z^{\prime} \in C[0,1]$ and

$$
\lim _{t \rightarrow 0^{+}} T(t) z^{\prime}(t)=R z^{\prime}(0)
$$

This lemma says that any component $z_{i}$ of $z$ in a block associated with $\alpha_{k} \geq 1$ satisfies $\lim _{t \rightarrow 0^{+}} t^{\alpha_{k}} z_{i}^{\prime}(t)=0$, while the components (if any) associated with $\alpha_{r}=0$ are in $C^{1}[0,1]$. This smoothness result can be extended if further restrictions are imposed on the problem.

Lemma 7 Assume that

(1) (N1)-(N5) hold.

(2) All $\alpha_{k}$ are integers.

(3) The real parts of the eigenvalues of the matrix $M_{k k}$ are negative whenever $\alpha_{k}=1$.

(4) $f \in C^{m}\left(T_{\rho}(z)\right)$.

Then, $z \in C^{m}[0,1] \cap C^{m+1}(0,1]$.

\section{Pathfollowing}

With the prerequisites from $\S 2$ we are in a position to describe our pathfollowing strategy for (1), (2) and formulate sufficient conditions for the procedure 
to be well-defined. We will first discuss our problem in a general framework and subsequently specialize the results to singular boundary value problems. Hence, consider (1), (2) as a parameter-dependent operator equation

$$
F(y ; \lambda)=0
$$

where $F: \hat{Y}:=Y \times \mathbb{R} \rightarrow Z$, and $Y, Z$ are Banach spaces (of possibly infinite dimension). If $\|\cdot\|$ denotes the norm on $Y$, then $\|(y, \lambda)\|_{\hat{Y}}^{2}:=\|y\|^{2}+\lambda^{2}$ is a norm on $\hat{Y}$. The norm on $Z$ is denoted by $\|\cdot\|_{Z}$.

Pathfollowing in this general setting has been discussed in detail in [5]. The following presentation is mainly based on these results. For proofs of our propositions we refer the reader to [5].

We require henceforth that the problem satisfies the following assumptions:

(A1) Problem (27) has a smooth solution branch $\Gamma$ which can be parametrized by a parameter ${ }^{2} s$,

$$
\Gamma:=\{(y(s), \lambda(s)): s \in[a, b]\}, \quad \text { where } \quad \frac{d}{d s}(y(s), \lambda(s)) \neq 0 \quad \forall s \in[a, b] .
$$

(A2) $F$ is continuously differentiable in a neighborhood

$$
T_{\rho}(\Gamma):=\left\{(y(s), \lambda(s))+(v, \eta): s \in[a, b],\|(v, \eta)\|_{\hat{Y}} \leq \rho\right\} .
$$

Moreover, the total derivative $F^{\prime}$ is bounded and Lipschitz-continuous on $T_{\rho}(\Gamma)$.

(A3) The partial derivative with respect to $y, F_{y}(y ; \lambda)$, is a Fredholm operator with index 0 for all $(y, \lambda) \in \Gamma$.

(A4) For all $(y, \lambda) \in \Gamma$, the nullspace of $F^{\prime}(y ; \lambda)$ has dimension 1 , or equivalenly, the range of $F^{\prime}(y ; \lambda)$ is equal to the whole space $Z$.

We are particularly interested in computing solution branches with turning points. By definition, in a turning point the solution of (27) constitutes a local maximum (or minimum) of $\lambda$, and consequently is not locally unique as a function of the parameter $\lambda$. The situation is illustrated in Figure 1. There, we plot some functional of the solution in dependence of the parameter $\lambda$. The arrows indicate the turning points. Thus, in a turning point we cannot parametrize $\Gamma$ as a function of $\lambda$. However, it is sufficient for our procedure that a tangent is uniquely determined at all points of $\Gamma$. This is guaranteed by our assumptions (A1)-(A4). Moreover, from (A1)-(A4) we may conclude that every turning point is a weak singularity of the solution branch. At a weak

$\overline{2}$ Typically, the arclength of the solution branch could be used in the situations we have in mind. 
singularity $\left(y^{*}, \lambda^{*}\right)$, the dimension of the nullspace of $F_{y}\left(y^{*} ; \lambda^{*}\right)$ is equal to 1 and the partial derivative $F_{\lambda}\left(y^{*} ; \lambda^{*}\right)$ does not lie in the range of $F_{y}\left(y^{*} ; \lambda^{*}\right)$. Thus, the weak singularities comprise turning points and points where the solution is locally unique as a function of $\lambda$, but no other types of bifurcation points. Subsequently, we will consider solution branches $\Gamma$ which contain at most weak singularities.

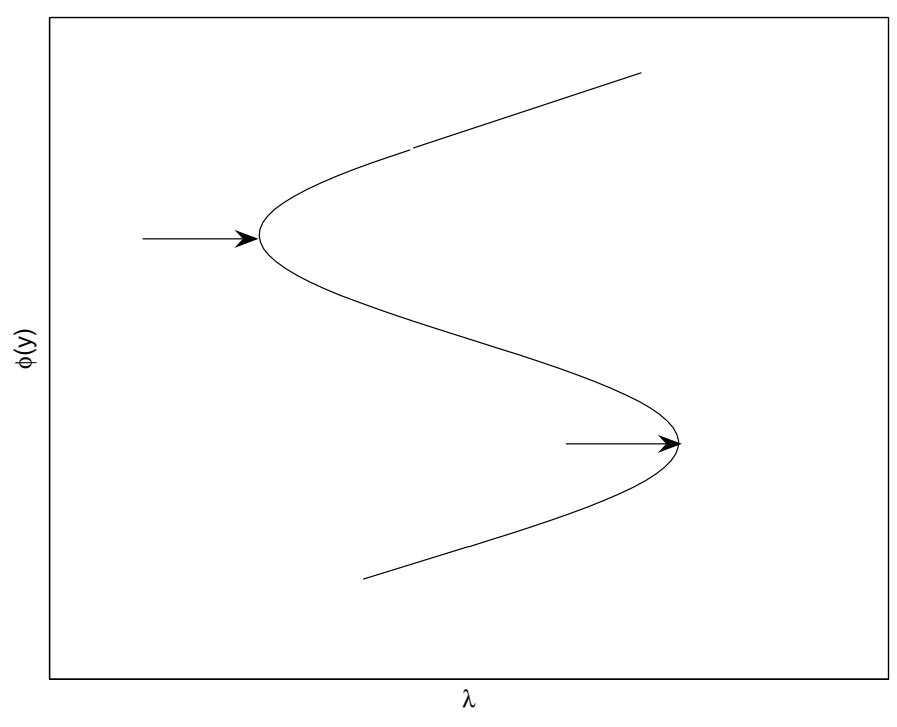

Fig. 1. A solution branch with two turning points.

In the definition and the analysis of our pathfollowing strategy, we require a continuous imbedding of the Banach space $Y$ into a Hilbert space $H$. Thus, we assume

(A5) Let $H$ be a Hilbert space with inner product $\langle\cdot \mid \cdot\rangle$ and induced norm $\|\cdot\|_{H}$, and for the Banach space $Y$ with norm $\|\cdot\|$ let $Y \subseteq H$ and $\|y\|_{H} \leq \mathcal{C}\|y\| \forall y \in Y$.

Note that this also induces a continuous imbedding of the Banach space $\hat{Y}$ into $\hat{H}:=H \times \mathbb{R}$. Here, $\langle(y, \lambda) \mid(v, \eta)\rangle_{\hat{H}}:=\langle y \mid v\rangle+\lambda \eta$ defines the inner product of $\hat{H}$ with induced norm $\|\cdot\|_{\hat{H}}$. Moreover, the estimate

$$
\|(y, \lambda)\|_{\hat{H}} \leq \max \{1, \mathcal{C}\}\|(y, \lambda)\|_{\hat{Y}}
$$

holds.

Now, we proceed by describing our pathfollowing strategy. As explained above, our assumptions ensure that at a point $\left(y_{0}, \lambda_{0}\right) \in \Gamma$, a tangent $(\delta y, \delta \lambda)$ can be 
determined from

$$
F_{y}\left(y_{0} ; \lambda_{0}\right) \delta y+F_{\lambda}\left(y_{0} ; \lambda_{0}\right) \delta \lambda=0
$$

In Theorem 8 below we are going to show that if we require

$$
\|(\delta y, \delta \lambda)\|_{\hat{H}}^{2}=1
$$

the tangent vector is uniquely determined up to the sign. We will explain how to choose the direction below. On the tangent just determined, we now choose a predictor for the computation of the next point on $\Gamma$,

$$
\left(y_{P}, \lambda_{P}\right):=\left(y_{0}, \lambda_{0}\right)+h(\delta y, \delta \lambda)
$$

with a suitable step-size $h>0$. Now, to compute the next point $\left(y_{C}, \lambda_{C}\right)$ on $\Gamma$, we define a linear functional

$$
\ell(y, \lambda):=\langle(\delta y, \delta \lambda) \mid(y, \lambda)\rangle_{\hat{H}}
$$

and subsequently solve the corrector equation

$$
G\left(y_{C}, \lambda_{C}\right):=\left(\begin{array}{c}
F\left(y_{C} ; \lambda_{C}\right) \\
\ell\left(y_{C}, \lambda_{C}\right)-\ell\left(y_{0}, \lambda_{0}\right)-h
\end{array}\right)=0
$$

where the predictor $\left(y_{P}, \lambda_{P}\right)$ serves as an initial approximation for the solution of this nonlinear system. Note that (33) is equivalent to the requirement that $\left(y_{C}, \lambda_{C}\right) \in \Gamma$ and the correction $\left(y_{C}, \lambda_{C}\right)-\left(y_{P}, \lambda_{P}\right)$ is orthogonal to the tangent vector $(\delta y, \delta \lambda)$. One step of our procedure starting at $\left(y_{0}, \lambda_{0}\right)$ is illustrated in Figure 2.

To ensure that our pathfollowing procedure advances in the right direction, and does not turn back at some point during our computation, we have to define a criterion for the choice of the direction of the tangent vector. It turns out that the requirement

$$
\ell\left(\delta y_{0}, \delta \lambda_{0}\right)=\left\langle(\delta y, \delta \lambda) \mid\left(\delta y_{0}, \delta \lambda_{0}\right)\right\rangle_{\hat{H}}>0
$$

serves this purpose, where $\left(\delta y_{0}, \delta \lambda_{0}\right)$ is the tangent vector used in the previous step of the pathfollowing procedure.

Note that with assumption (A5) and the definition (32) we may conclude that

$$
\|\ell\|_{\hat{Y}^{\prime}} \leq C_{1},
$$




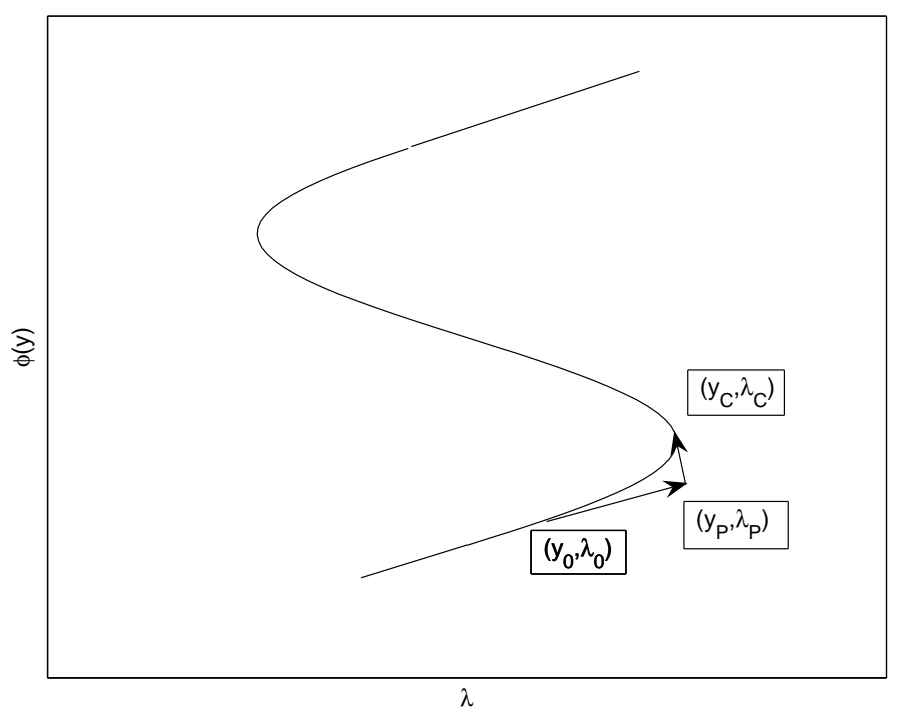

Fig. 2. One step of the pathfollowing procedure.

where $\|\cdot\|_{\hat{Y}^{\prime}}$ denotes the norm on the dual space of $\hat{Y}$.

Next, we will demonstrate that the equations (29), (30) and (33) defining our pathfollowing procedure are isolatedly solvable and well-posed. To this end, we quote the following results proven in [5], see also [6]:

Theorem 8 Assume that (A1)-(A5) hold. Then there exist positive constants $\rho, \varepsilon, h_{0}$, such that the following assertions hold for all $s \in[a, b],(y, \lambda) \in$ $T_{\rho}(\Gamma), h \leq h_{0}$ and $(v, \eta) \in Z \times \mathbb{R}$ with $\|v\|_{Z}+\eta^{2} \leq \varepsilon$ :

(1) The equation

$$
\left(\begin{array}{c}
F_{y}(y, \lambda) \delta y+F_{\lambda}(y, \lambda) \delta \lambda \\
\|(\delta y, \delta \lambda)\|_{\hat{H}}^{2}-1
\end{array}\right)=0
$$

has a solution which is unique except for the sign and depends Lipschitzcontinuously on $(y, \lambda)$.

(2) The equation

$$
G\left(y_{C}, \lambda_{C}\right)=\left(\begin{array}{c}
F\left(y_{C}, \lambda_{C}\right) \\
\ell\left(y_{C}, \lambda_{C}\right)-\ell\left(y_{0}, \lambda_{0}\right)-h
\end{array}\right)=\left(\begin{array}{l}
v \\
\eta
\end{array}\right)
$$

has an isolated solution if the functional $\ell$ satisfies

$$
|\ell(\delta y, \delta \lambda)| \geq c_{1}\|(\delta y, \delta \lambda)\|_{\hat{Y}}
$$


with a constant $0<c_{1} \leq C_{1}$, cf. (35). The inverse of the linearization of $G$ at $\left(y_{C}, \lambda_{C}\right)$ is uniformly bounded.

(3) If $\|(y, \lambda)-(y(s), \lambda(s))\|_{\hat{Y}} \leq \rho$ for some $a<s<b$, then $\left(y_{C}, \lambda_{C}\right) \in T_{\rho}(\Gamma)$.

Now, we reformulate the assumptions necessary for the last theorem to hold in terms of our singular boundary value problem (1), (2).

For simplicity, assume here that our problem has the form

$$
\begin{aligned}
& T(t) z^{\prime}(t)=f(t, z(t) ; \lambda):=(M+A(t)) z(t)+T(t) \hat{f}(t, z(t) ; \lambda), \\
& b(z(0), z(1))=0,
\end{aligned}
$$

with $T(t), M$ defined in (6) and (10), respectively, and the continuity requirement incorporated into $b$ in the form (21). In this case, for all $(z, \lambda) \in \Gamma$ we can define the operator $\mathcal{L}$ analogously as in $\S 2.1$ using the differential expression $l$ from (18). Now, $Y$ is the Banach space of all $z \in C[0,1]$ for which $T z^{\prime} \in C[0,1]$, and $Z=C[0,1] \times \mathbb{R}^{n}$. Assumption (A5) holds, since $Y$ can be continuously imbedded into the Hilbert space $L^{2}(0,1)$, and

$$
\langle y \mid y\rangle=\int_{0}^{1}|y(t)|^{2} d t \leq\|y\|_{\infty}^{2} \leq\|y\|_{\mathcal{L}}^{2} .
$$

We assume that the parameter-dependent problem (39), (40) satisfies (A1).

(A2) holds if the right-hand side $f$ of (39) is continuously differentiable w.r.t. $z, \lambda$ on

$$
\left\{(t, z(t ; s), \lambda(s))+(0, v, \eta): s \in[a, b], t \in[0,1],|v| \leq \rho_{0},|\lambda| \leq \rho_{0}\right\},
$$

and $b$ from (40) is continuously differentiable w. r.t. both variables on

$$
\left\{(z(0 ; s), z(1 ; s))+\left(v_{1}, v_{2}\right): s \in[a, b],\left|v_{1}\right| \leq \rho_{0},\left|v_{2}\right| \leq \rho_{0}\right\}
$$

and these partial derivatives of $f, b$ are bounded and Lipschitz-continuous for some $\rho_{0}>0$.

(A3) holds if the linearization of the problem (39), (40) w.r.t. $z$ (see (22)$(24))$ at $(z, \lambda) \in \Gamma$ is Fredholm with index 0. Sufficient conditions for a singular boundary value problem to have this property have been given in Theorem 3 and Theorem 4.

Finally, (A4) is satisfied if for all $(z, \lambda) \in \Gamma$ and all $(g, \gamma) \in Z$ the linear problem

$$
T(t) v^{\prime}(t)-G(t) v(t)-c(t) \eta=g(t)
$$




$$
B_{0} v(0)+B_{1} v(1)=\gamma
$$

with $G, B_{0}, B_{1}$ defined in $(25)$ and $c(t):=f_{\lambda}(t, z(t) ; \lambda)$, has a solution $(v, \eta) \in$ $\hat{Y}$. If we rewrite this problem by letting $\eta=\eta(t)$ and add the trivial equation $\eta^{\prime}(t)=0$, this equation can be analyzed within the framework given in $\S 2.1$.

\subsection{Numerical Solution}

Here, we briefly discuss the numerical realization of the pathfollowing strategy described above. As the basic solution method, we use polynomial collocation. It has been demonstrated in [10] that this method is well suited for the solution of boundary value problems with an essential singularity and rapidly convergent for this problem class, see also [1], [11], [12]. Our Matlab implementation is designed for the solution of boundary value problems of mixed order (also enabling the treatment of purely algebraic equations and solution for unknown parameters) in an implicit formulation. To enhance the efficiency of the solver, we have added an adaptive mesh selection strategy aiming at the equidistribution of the global error, which is approximated by an asymptotically correct error estimate. For a description of the collocation code and a large number of numerical examples, see [2]. The basic principles of our collocation solver, error estimate and mesh adaptation are described for example in [10], [13], [14], and [15]. We do not go into details of our numerical solution routines here.

Now, assume that we have already computed a point $\left(y_{\Delta}, \lambda_{\Delta}\right) \approx(y(s), \lambda(s))$ on a mesh $\Delta$, which is sufficiently close to $\Gamma$ for our desired accuracy (as guaranteed by our a posteriori error estimation procedure and adaptive mesh selection). Then, due to Theorem 8 the linear equation (36) has a unique solution. If we discretize (36) on the mesh $\Delta$, this represents a set of collocation equations with an additional algebraic constraint, which can be treated by our solver, thus yielding an approximation $\left(\delta y_{\Delta}, \delta \lambda_{\Delta}\right)$ to the tangent. Subsequently, we can solve (33) on the same mesh $\Delta$. Since the solution of (33) is isolated, this can again be computed numerically if the initial approximation is good enough. This is the case if the tangent direction is sufficiently accurate and the step-size $h$ used to define the predictor (31) is small enough. In the solution of (33), we have to ensure sufficient accuracy by adapting the mesh according to our tolerances. Note that whenever the mesh is refined, the tangent direction should be recomputed on the new mesh. This poses no problem, since the data $\left(y_{\Delta}, \lambda_{\Delta}\right)$ used to define (36) can be evaluated at arbitrary points. To recapitulate, due to the results given in Theorem 8 it is possible to implement our pathfollowing procedure based on our collocation solver, where the computational problems are well-defined if the computations are performed with sufficient accuracy and the step-size used for the predictor 
points $\left(y_{P}, \lambda_{P}\right)$ is small enough. Moreover, the computational problems are well-posed due to Theorem 8.

\section{Numerical Results}

As a first example to demonstrate that our pathfollowing procedure works reliably in conjunction with the integrated mesh-adaptation, we compute a solution branch for a well-known, regular problem from chemistry which describes the catalytic reaction in a flat particle [16],

$$
\begin{aligned}
& z^{\prime \prime}(t)=\lambda z(t) \exp \left(\frac{8(1-z(t))}{1+0.4(1-z(t))}\right), \quad t \in[0,1] \\
& z^{\prime}(0)=0, \quad z(1)=1
\end{aligned}
$$

We start for $\lambda=0$ at a solution computed on a grid containing 11 equidistant mesh points and three Gaussian collocation points in each subinterval. Our goal is to compute a solution branch $\Gamma$ with two turning points, see Figure 3. At each point of $\Gamma$, we prescribe a mixed error tolerance with absolute and relative tolerance $1 \mathrm{e}-6$. The mesh is adapted automatically in each step, where both an increase and a decrease in the number of points is possible according to the difficulty of meeting the tolerance requirement. In Figure 3 we show the value of $z(0)$ in dependence of the parameter $\lambda$. At the points marked with a cross in Figure 3, we plot the solution profile $z(t)$ and the mesh automatically generated by our adaptive procedure in Figure 4. We observe that the meshes seem to be reasonably adapted to the difficulty of the solution, which features an increasingly steep layer as the computation proceeds.

To demonstrate that indeed our pathfollowing strategy also works for singular boundary value problems and generates meshes adapted to the solution profile, we consider an example from [17],

$$
\begin{aligned}
& \delta\left(\beta^{\prime \prime}(t)+\beta^{\prime}(t) \cot (t)+\cot ^{2} t \frac{\cos (t-\beta(t))}{\cos t} \frac{\sin (t-\beta(t))-\sin t}{\cos t}\right. \\
& \left.-0.3 \frac{\cos (t-\beta(t))-\cos t}{\sin t}\right) \\
& =-\Psi(t) \frac{\sin (t-\beta(t))}{\sin t}-4 \lambda \frac{\cos (t-\beta(t))}{\sin t} \int_{0}^{t} \cos (\eta-\beta(\eta)) \sin \eta d \eta, \\
& \delta\left(\Psi^{\prime \prime}(t)+\Psi^{\prime}(t) \cot t-\Psi(t)\left(\cot ^{2}(t) \frac{\cos ^{2}(t-\beta(t))}{\cos ^{2} t}\right.\right.
\end{aligned}
$$




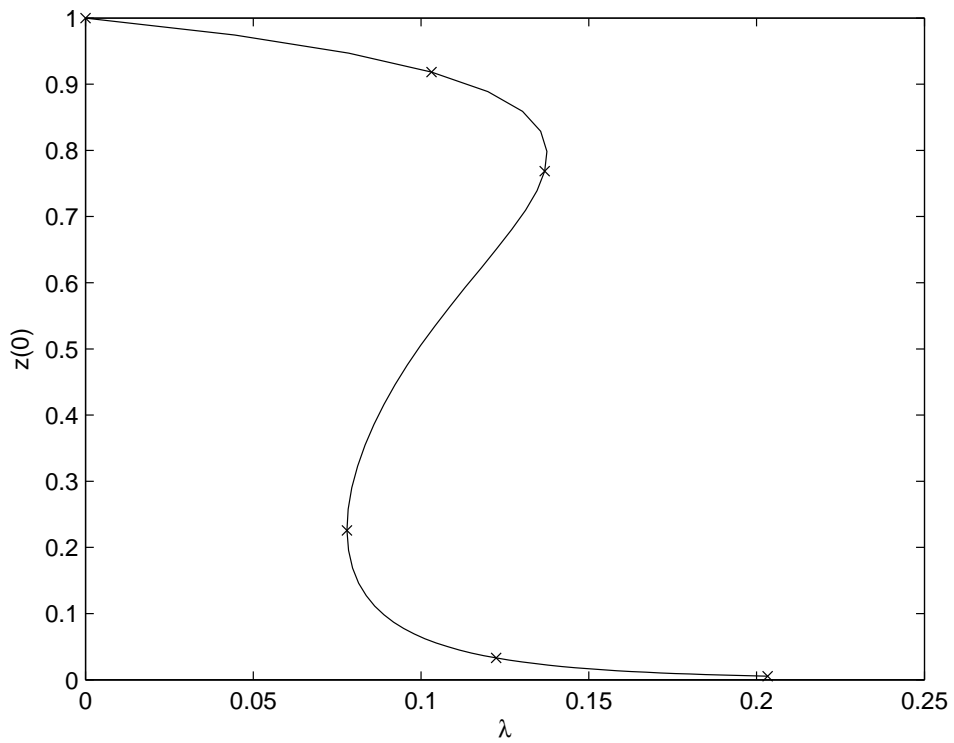

Fig. 3. Values of $z(0)$ along a solution branch of (41), (42).
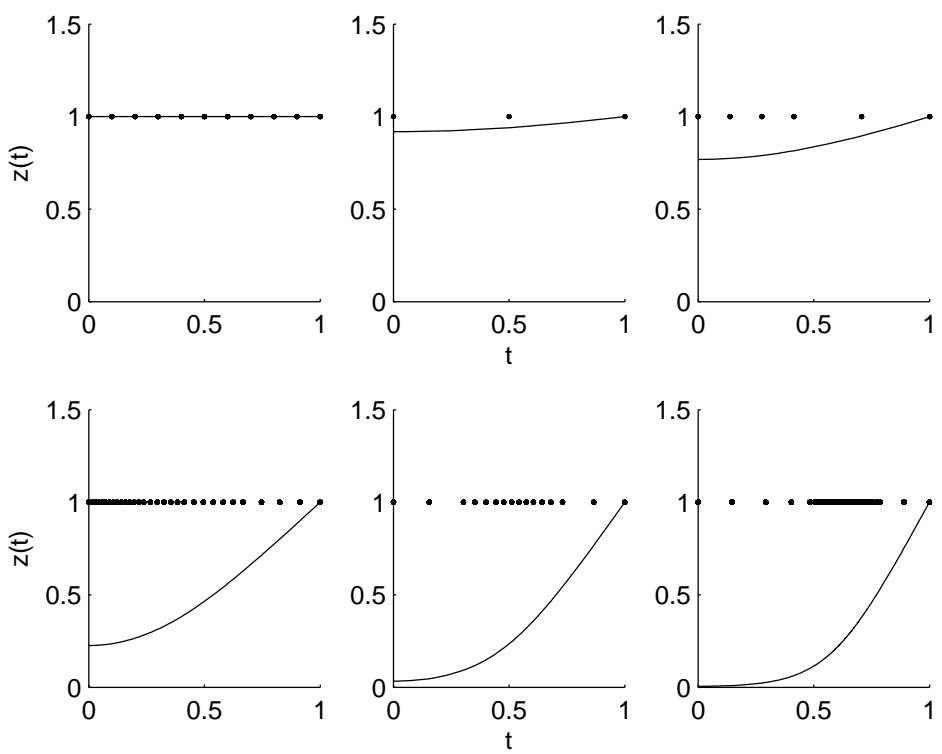

Fig. 4. Solution profiles and automatically selected meshes at the points marked in Figure 3 along the solution branch of (41), (42).

$$
\begin{aligned}
& \left.\left.-0.3\left(1-\beta^{\prime}(t)\right) \frac{\sin (t-\beta(t))}{\sin t}\right)\right) \\
= & \frac{\cos (t-\beta(t))-\cos t}{\sin t}+\delta\left(-4 \lambda \cot t \int_{0}^{t} \cos (\eta-\beta(\eta)) \sin \eta \mathrm{d} \eta\right.
\end{aligned}
$$




$$
\begin{aligned}
& \cdot\left(\frac{\sin (2(t-\beta(t)))}{\sin (2 t)}+0.3\left(1-\beta^{\prime}(t)\right) \frac{\cos (t-\beta(t))}{\cos t}\right) \\
& \left.+4 \lambda \frac{\left(\sin ^{2} t \sin (t-\beta(t))\right)^{\prime}}{\sin t}\right),
\end{aligned}
$$

where $\delta=0.00369$. The boundary conditions are

$$
\beta(0)=\beta(\pi)=0, \quad \Psi(0)=\Psi(\pi)=0 .
$$

To treat the integral occurring in (43) and (44) in a form suitable for our code, we introduce the auxiliary variable

$$
i(t)=\int_{0}^{t} \cos (\eta-\beta(\eta)) \sin \eta \mathrm{d} \eta
$$

and augment our system with the equations

$$
\begin{aligned}
i^{\prime}(t) & =\cos (t-\beta(t)) \sin t, \\
i(0) & =0 .
\end{aligned}
$$

We follow the path $\Gamma$ shown in Figure 5, starting at $\lambda=0$. For numerical reasons it was necessary to choose a fixed, uniform mesh (stepsize $=0.01,5$ Gaussian points) to pass the first turning point, because the desired solution branch is very easy to miss in that regime. At $\lambda \approx 0.6$ the strategy is changed and an absolute and relative tolerance of $1 \mathrm{e}-8$ is prescribed, whence the adaptive mesh refinement can be used satisfactorily to produce meshes adapted to the solution behavior. Figure 5 shows the maximum norm of the first solution component, $\|\beta\|_{\infty}$ along the path $\Gamma$. The crosses indicate points of $\Gamma$ where the solution profiles of $\beta$ and $\Psi$ are plotted in Figure 6, together with the meshes generated by our adaptive mesh selection procedure. A comparison with [17, Figure 10] shows that the solution is computed reliably and obviously the meshes are denser where the solution varies more strongly.

\section{The Complex Ginzburg-Landau Equation}

As a further numerical example to demonstrate that our pathfollowing strategy can be successfully applied to practically relevant problems, we consider the complex Ginzburg-Landau equation (CGL) 


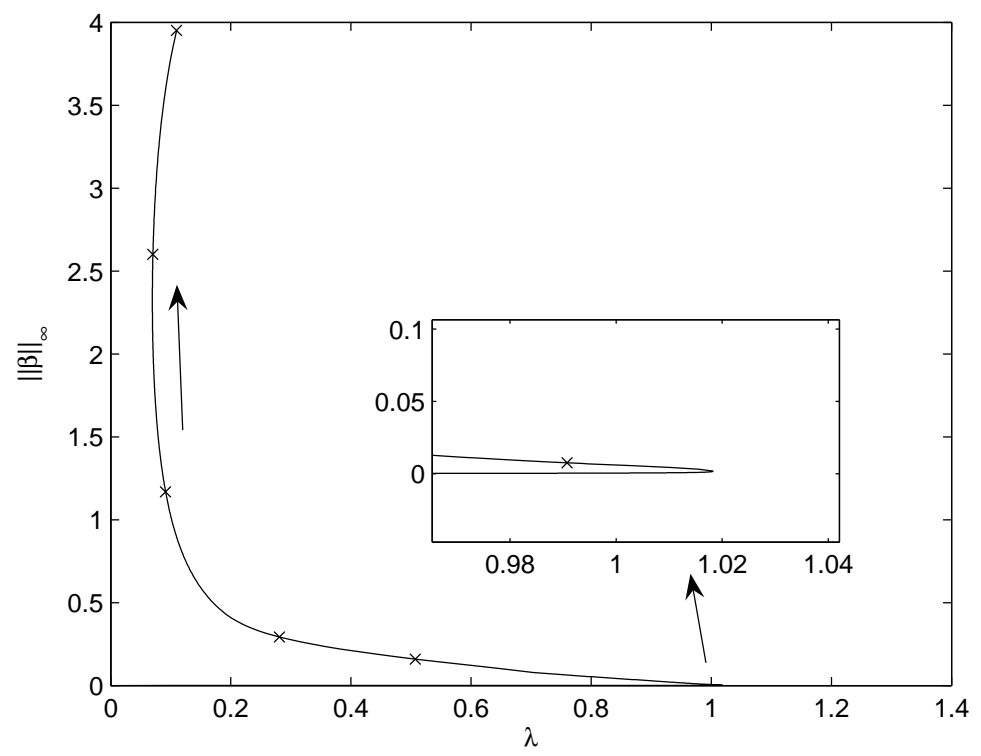

Fig. 5. Values of $\|\beta\|$ along a solution branch of (43)-(47).

$$
\begin{aligned}
& \mathrm{i} \frac{\partial u}{\partial t}+(1-\mathrm{i} \varepsilon) \Delta u+(1+\mathrm{i} \delta)|u|^{2} u=0, \quad t>0, \\
& u(x, 0)=u_{0}(x), \quad x \in \mathbb{R}^{d}
\end{aligned}
$$

which arises as a model in a variety of problems from physics, biology and chemistry. These include nonlinear optics, models of turbulence, RayleighBenard convection, superconductivity, superfluidity, Taylor-Couette flow and reaction-diffusion systems, see for example [18]. Henceforth, we will consider the case $d=3$ and restrict ourselves to radially symmetric solutions.

Equation (48) is a perturbed version of the nonlinear Schrödinger equation $(N L S)$ which takes the same form with $\varepsilon=\delta=0$. It has been conjectured that both equations have blow-up solutions which become singular in finite time and obey the same scaling laws as the differential equation, see [7], [8], [19], [20]. After a similarity reduction (see for example [1], [7], [11]), the selfsimilar solution profile can be computed from the nonlinear ODE for $\tau>0$,

$$
(1-\mathrm{i} \varepsilon)\left(y^{\prime \prime}(\tau)+\frac{2}{\tau} y^{\prime}(\tau)\right)-y(\tau)+\mathrm{i} a(\tau y(\tau))^{\prime}+(1+\mathrm{i} \delta)|y(\tau)|^{2} y(\tau)=0,
$$

with the boundary conditions

$$
y^{\prime}(0)=0, \quad \Im y(0)=0, \quad \lim _{\tau \rightarrow \infty} \tau y^{\prime}(\tau)=0 .
$$

In addition to the solution function $y$, the unknown (non-negative) parameter $a$ is also to be determined from this system. It was demonstrated in [8], [19] 

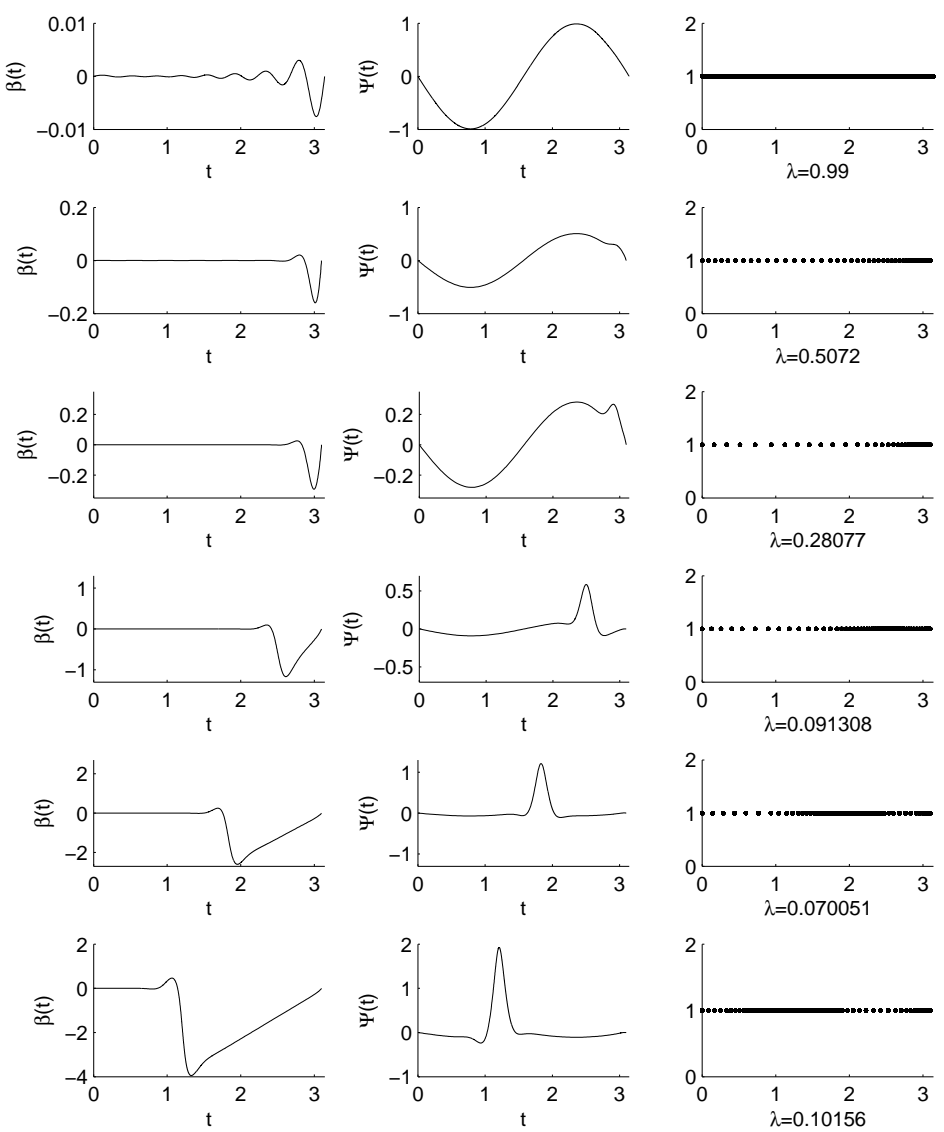

Fig. 6. Solution profiles and automatically selected meshes at the points marked in Figure 5 along the solution branch of (43)-(47).

that the problem (50), (51) corresponding to the NLS has a stable, monotone solution and a plethora of unstable non-monotone ("multi-bump") solutions. It was found that the CGL has corresponding solution branches when the perturbation parameters $\varepsilon, \delta$ are varied, see [7]. In addition, stable non-monotone solutions exist which can be obtained by pathfollowing past a turning point, see [7] and [20]. We will demonstrate that a transformation of (50) to a boundary value problem with an essential singularity on the interval $[0,1]$ and solution by our Matlab implementation of the pathfollowing strategy described in $\S 3$ can serve to successfully compute the solution branches across the turning points.

In [2], we use transformations similarly as in [1], [11] to derive the singular 
boundary value problem ${ }^{3}$

$$
z^{\prime}(\tau)=\left(\begin{array}{cc}
\frac{M(\tau)}{\tau} & 0 \\
0 & \frac{A(\tau)}{\tau^{3}}
\end{array}\right) z(\tau)+\left(\begin{array}{c}
f\left(\tau, z_{1}(\tau), z_{2}(\tau)\right) \\
g\left(\tau, z_{3}(\tau), z_{4}(\tau)\right)
\end{array}\right)
$$

where

$$
\begin{aligned}
& M(\tau)=\left(\begin{array}{cc}
0 & \tau \\
\tau \frac{1+\varepsilon a+\mathrm{i}(\varepsilon-a)}{1+\varepsilon^{2}} & -2+\tau \frac{\varepsilon a-\mathrm{i} a}{1+\varepsilon^{2}}
\end{array}\right), \\
& f(\tau, z)=\left(\begin{array}{c}
0 \\
\frac{\varepsilon \delta-1-\mathrm{i}(\varepsilon+\delta)}{1+\varepsilon^{2}} z_{1}\left|z_{1}\right|^{2}
\end{array}\right), \\
& A(\tau)=\left(\begin{array}{cc}
0 & -\tau^{2} \\
-\frac{1+\varepsilon a+\mathrm{i}(\varepsilon-a)}{1+\varepsilon^{2}} & \frac{\mathrm{i} a-\varepsilon a}{1+\varepsilon^{2}}+\tau^{2}
\end{array}\right) \text {, } \\
& g\left(\tau, z_{3}, z_{4}\right)=\left(\begin{array}{c}
0 \\
-\frac{1}{\tau^{3}} \frac{\varepsilon \delta-1-\mathrm{i}(\varepsilon+\delta)}{1+\varepsilon^{2}} z_{3}\left|z_{3}\right|^{2}
\end{array}\right) .
\end{aligned}
$$

Additionally, we prescribe the boundary conditions

$$
z_{2}(0)=0, \Im z_{1}(0)=0, z_{1}(1)=z_{3}(1), z_{2}(1)=z_{4}(1), z_{4}(0)=0 .
$$

In order to discuss the well-posedness of the resulting singular boundary value problem, we have to consider the spectrum of the matrices $M(0)$ and $A(0)$, see [3], [9]. The same type of analysis was performed for the NLS in [1] and [11]. There, it was found that (53) constitute necessary and sufficient conditions for the existence of an isolated, continuous solution of the problem. For $\varepsilon \neq 0$, the analysis proceeds similarly: The eigenvalues of $M(0)$ are $\lambda_{1}=0$ and $\lambda_{2}=-2$. Thus, the condition $z_{2}(0)=0$ is necessary and sufficient for a continuous solution to exist. The eigenvalues of $A(0)$ are $\lambda_{3}=0$ and $\lambda_{4}=\frac{i a-\varepsilon a}{1+\varepsilon^{2}}$. Since the latter has a negative real part for $\varepsilon \neq 0, z_{4}(0)=0$ is an admissible boundary condition. Altogether, we conclude that the boundary conditions (53) yield a well-posed boundary value problem for (52).

Now, we give some numerical results computed by our pathfollowing strategy described in $\S 3$. A full account of our experiments is given in [2]. Due to the

$\overline{3}$ In [1], [11] a slightly different transformation was used which resulted in a different matrix $M(\tau)$. Since the eigenstructure of $M(0)$ remains unaltered by our modification, all conclusions remain valid. 
difficulty of the problem, we restricted ourselves to fixed meshes adapted to the solution profile in order to obtain satisfactory results. First, we consider the case where $\delta=0$ and $\varepsilon$ is varied. This case is also treated in [7], so a comparison can serve to validate our results. Figure 7 shows the values of the unknown parameter $a$ and of $y(0)=z_{1}(0)$ along a solution branch in dependence of $\varepsilon$. The branch bifurcates from a 'multi-bump' solution of the NLS, which is also computed in [8] and referred to as the ' $(0,1)$ solution branch' there. The results correspond well with [7, Figure 1.1]. The turning point was numerically found to be located at approximately $\varepsilon=0.24359$, which also agrees with the results from [7].
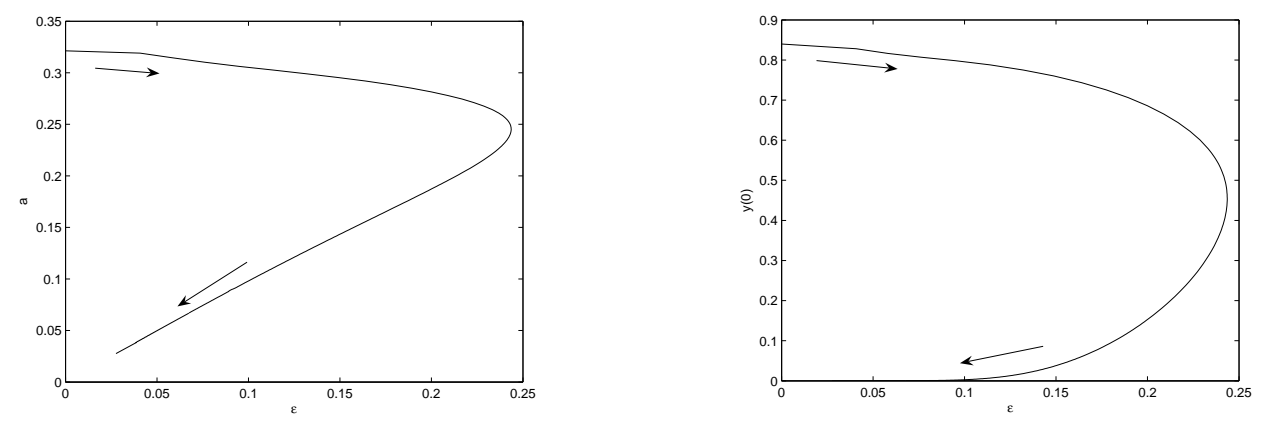

Fig. 7. Values of $a$ (left) and $y(0)$ (right) along a branch bifurcating from the '(0,1) solution branch' of the NLS.

More interestingly, our method could also serve to compute solution branches not discussed in the literature. Figure 8 shows the results obtained when starting from the ' $(1,3)$ ' solution of the NLS. This branch has a more intricate profile, the value of $a$ apparently features three turning points when considered as a function of $\varepsilon$ and is non-monotonous along the branch. This is no contradiction, however, since the profile of $y(0)$ shows that the intersections in the graph for $a$ do not actually represent bifurcation points. Here, we encounter an intriguing phenomenon: By following the present solution branch back to the value $\varepsilon=0$, we obtain a real-valued solution of the NLS, where $a=0$. This solution profile is given in Figure 9.
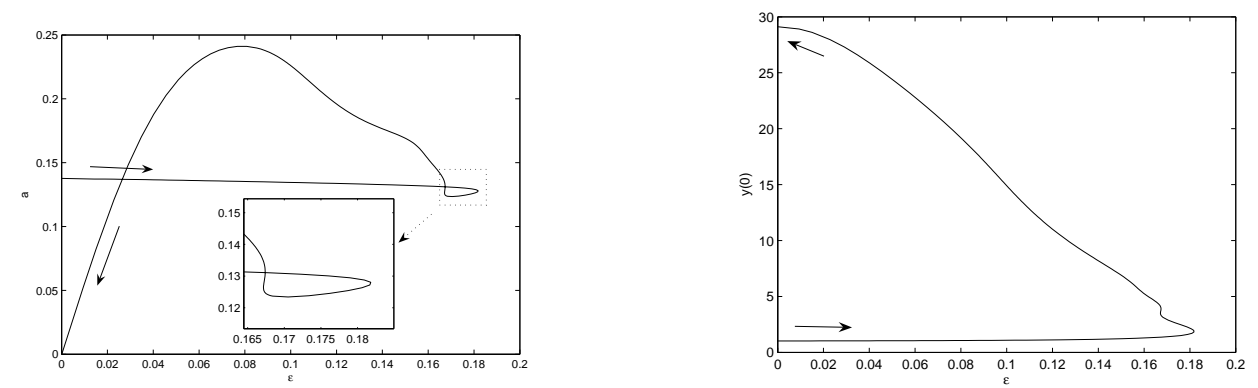

Fig. 8. Values of $a$ (left) and $y(0)$ (right) along a branch bifurcating from the '(1,3) solution branch' of the NLS.

To conclude the discussion of the complex Ginzburg-Landau equation, we 


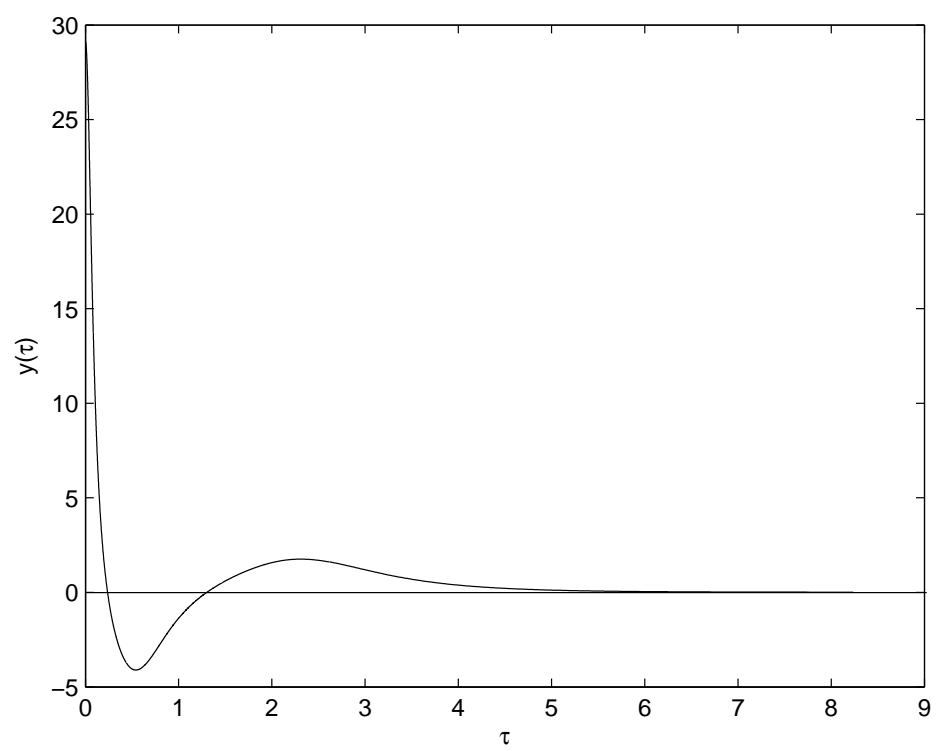

Fig. 9. Real solution of the NLS computed by pathfollowing starting from the ' $(1,3)$ solution branch'.

show that our solution method is also useful in determining solution behavior in situations which to our knowledge have not yet been treated in the literature. We set $\delta=\varepsilon$ and compute solution branches in dependence of this new parameter. The results obtained when starting from the ' $(0,1)$ ' solution of the NLS are given in Figure 10. The qualitative behavior is similar as in the case $\delta=0$. However, the turning point is encountered for smaller values of $\varepsilon$, cf. Figure 7.
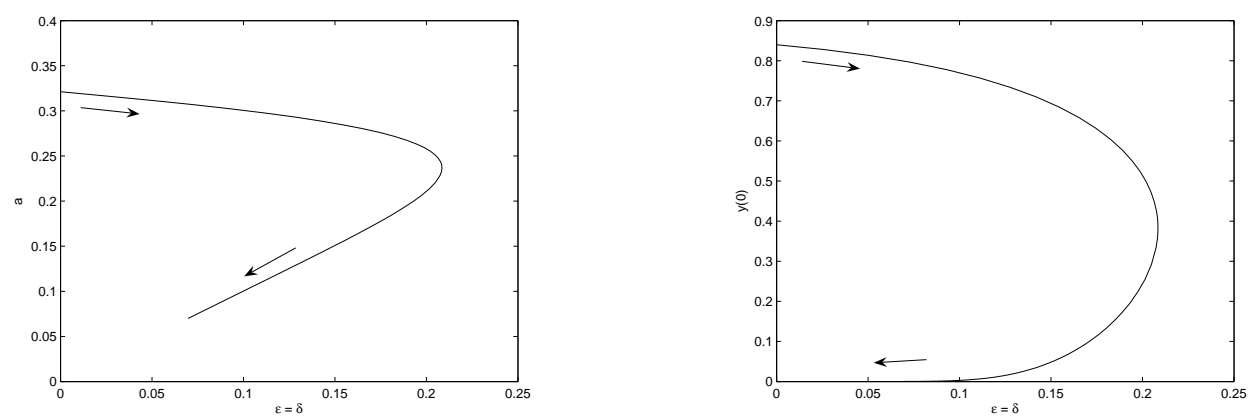

Fig. 10. Values of $a$ (left) and $y(0)$ (right) along a branch bifurcating from the ' $(0,1)$ solution branch' of the NLS, $\delta=\varepsilon$.

\section{Conclusions}

In this paper, we have discussed the application of a pathfollowing strategy based on pseudo-arclength parametrization to the solution of parameter- 
dependent singular boundary value problems. We could formulate necessary conditions for the well-posedness of the procedure when a singularity is present, and demonstrated that an implementation of the method based on polynomial collocation with adaptive mesh refinement could serve to solve practically relevant problems. Particularly, the computation of self-similar blow-up solutions of the complex Ginzburg-Landau equation was successful. This was illustrated by comparisons with results given in the literature, but also by computing solution branches in hitherto disregarded situations.

The advantages of our solution approach are manifold: The transformation to a finite interval enables to use efficient mesh selection strategies which automatically adapt the mesh according to an a posteriori error estimate. The solution routines used for the treatment of the singular boundary value problems are justified theoretically and experimentally. Our collocation solver can also be applied directly to problems of mixed order in an implicit formulation, which yields a favorable conditioning of the collocation equations [21]. Consequently, equations with an unknown parameter can also be solved effectively.

\section{References}

[1] C. J. Budd, O. Koch, E. Weinmüller, Self-similar blow-up in nonlinear PDEs, AURORA TR-2004-15, Inst. for Anal. and Sci. Comput., Vienna Univ. of Technology, Austria, available at http://www.vcpc.univie.ac.at/aurora/publications/ (2004).

[2] G. Kitzhofer, Numerical treatment of implicit singular BVPs, Ph.D. Thesis, Inst. for Anal. and Sci. Comput., Vienna Univ. of Technology, Austria, in preparation (2005).

[3] F. d. Hoog, R. Weiss, On the boundary value problem for systems of ordinary differential equations with a singularity of the second kind, SIAM J. Math. Anal. 11 (1980) 41-60.

[4] F. d. Hoog, R. Weiss, The numerical solution of boundary value problems with an essential singularity, SIAM J. Numer. Anal. 16 (1979) 637-669.

[5] R. Winkler, Path-following for two-point boundary value problems, Techn. Rep. 78, Department of Mathematics, Humboldt-University Berlin, Germany (1985).

[6] E. Weinmüller, R. Winkler, Pathfollowing algorithm for singular boundary value problems, ZAMM 68 (1988) 527-537.

[7] C. J. Budd, V. Rottschäfer, J. F. Williams, Multi-bump, blow-up, self-similar solutions of the complex Ginzburg-Landau equation, SIAM J. Appl. Dyn. Syst. 4 (2005) 649-678. 
[8] C. J. Budd, S. Chen, R. D. Russell, New self-similar solutions of the nonlinear Schrödinger equation with moving mesh computations, J. Comput. Phys. 152 (1999) 756-789.

[9] F. d. Hoog, R. Weiss, Difference methods for boundary value problems with a singularity of the first kind, SIAM J. Numer. Anal. 13 (1976) 775-813.

[10] W. Auzinger, O. Koch, E. Weinmüller, Collocation methods for boundary value problems with an essential singularity, in: I. Lirkov, S. Margenov, J. Wasniewski, P. Yalamov (Eds.), Large-Scale Scientific Computing, Vol. 2907 of Lecture Notes in Computer Science, Springer Verlag, 2004, pp. 347-354.

[11] C. J. Budd, O. Koch, E. Weinmüller, Computation of self-similar solution profiles for the nonlinear Schrödinger equation, to appear in Computing. Available at http://www.othmar-koch.org/research.html.

[12] C. J. Budd, O. Koch, E. Weinmüller, From nonlinear PDEs to singular ODEs, Appl. Numer. Math. 56 (2006) 413-422.

[13] W. Auzinger, O. Koch, E. Weinmüller, Efficient collocation schemes for singular boundary value problems, Numer. Algorithms 31 (2002) 5-25.

[14] W. Auzinger, O. Koch, D. Praetorius, E. Weinmüller, New a posteriori error estimates for singular boundary value problems, Numer. Algorithms 40 (2005) 79-100.

[15] W. Auzinger, O. Koch, E. Weinmüller, Efficient mesh selection for collocation methods applied to singular BVPs, J. Comput. Appl. Math. 180 (1) (2005) $213-227$.

[16] V. Hlavacek, M. Marek, M. Kubicek, Modelling of chemical reactors X. Multiple solutions of enthalpy and mass balances for a catalytic reaction within a porous catalyst particle, Chem. Eng. Sci. 23 (68) 1083-1097.

[17] M. Gräff, R. Scheidl, H. Troger, E. Weinmüller, An investigation of the complete post-buckling behavior of axisymmetric spherical shells, ZAMP 36 (1985) 803821.

[18] S. Aranson, I. L. Kramer, The world of the complex Ginzburg-Landau equation, Rev. Mod. Phys. 74 (2002) 99-143.

[19] C. J. Budd, Asymptotics of multibump blow-up self-similar solutions of the nonlinear Schrödinger equation, SIAM J. Appl. Math. 62 (3) (2001) 801-830.

[20] P. Plecháč, V. Šverák, On self-similar singular solutions of the complex Ginzburg-Landau equation, Comm. Pure Appl. Math. 54 (2001) 1215-1242.

[21] G. Kitzhofer, O. Koch, P. Lima, E. Weinmüller, Efficient numerical solution of the density profile equation in hydrodynamics, to appear in J. Sci. Comput. Available at http://www.othmar-koch.org/research.html (2005). 